ESAIM: PROCEEDINGS AND SURVEYS, September 2014, Vol. 45, p. 247-254

J.-S. Dhersin, Editor

\title{
METASTABILITY FOR SCALAR CONSERVATION LAWS IN A BOUNDED DOMAIN
}

\author{
Corrado Mascia $^{1,2}$ and Marta Strani ${ }^{3}$
}

\begin{abstract}
The initial-boundary-value problem for a viscous scalar conservation law in a bounded interval $I=(-\ell, \ell)$ is considered, with emphasis on the metastable dynamics, whereby the time-dependent solution develops internal transition layers that approach their steady state in an asymptotically exponentially long time interval as the viscosity coefficient $\varepsilon>0$ goes to zero. We describe such behavior by deriving an ODE for the position $\xi$ of the internal interface. The main tool of our analysis is the construction of a one-parameter family of approximate stationary solutions $\left\{U^{\varepsilon}(\cdot ; \xi)\right\}_{\xi \in I}$, parametrized by the location of the shock layer $\xi$, to be considered as an approximate invariant manifold for the problem. By using the properties of the linearized operator at $U^{\varepsilon}$, we estimate the size of the layer location.
\end{abstract}

\section{INTRODUCTION}

The slow motion of patterns of internal interfaces is a phenomenon known as metastability. The qualitative features of a metastable dynamics are the follows: through a transient process, a pattern of internal layers is formed from initial data in a $\mathcal{O}(1)$ time interval. Once this pattern is formed, the subsequent motion of the internal layers is exponentially slow, converging to their asymptotic limit. As a consequence, two different time scales emerge: for short times, the solutions are close to some non-stationary state; subsequently, they drifts towards the equilibrium solution with a speed rate that is exponentially small.

Slow motion of internal interfaces has been investigated for a number of different partial differential equations. To name just some of these results, we recall here [5], a pioneering article in the study of slow dynamics for viscous scalar conservation law; starting from this, there are several papers concerning slow motion of internal shock layer for viscous conservation law, see for example [6], [7], [10]. Slow dynamics of interfaces has been examined also for Burgers type equation (see [2] and [12] ), for relaxation system, with the contribution [11], and for phase transition problem described by the Allen-Cahn and Cahn-Hilliard equation in $[1,3,4,9]$.

In this paper we present some results contained in [8]. In particular, we apply the strategy firstly performed in [8] to rigorous describe the metastable behavior of solutions to the initial-boundary value problem for a scalar conservation law with viscosity with Dirichlet boundary condition in the bounded interval $I=(-\ell, \ell)$. In this specific case it is possible to obtain an explicit expression for the speed rate of convergence of the interface, showing how its motion in indeed exponentially slow.

1 Dipartimento di Matematica "G. Castelnuovo", Sapienza - Università di Roma, P.le Aldo Moro, 2 - 00185 Roma (ITALY), mascia@mat.uniroma1.it

${ }^{2}$ Istituto per le Applicazioni del Calcolo, Consiglio Nazionale delle Ricerche (associated in the framework of the program "Intracellular Signalling")

${ }^{3}$ Ecole Normale Supérieure, Département de Matéhmatiques et Applications, 45 rue d’Ulm - 75005 Paris (France), strani@dma.ens.fr

(C) EDP Sciences, SMAI 2014 
Let us consider

$$
\left\{\begin{aligned}
\partial_{t} u+\partial_{x} f(u) & =\varepsilon \partial_{x}^{2} u & & (x, t) \in I \times(0,+\infty) \\
u( \pm \ell, t) & =u_{ \pm} & & t>0 \\
u(x, 0) & =u_{0}(x) & & x \in I
\end{aligned}\right.
$$

for some $\varepsilon, \ell>0, u_{ \pm} \in \mathbb{R}$ and flux function $f$ satisfying

$$
f^{\prime \prime}(s) \geq c_{0}>0, \quad f^{\prime}\left(u_{+}\right)<0<f^{\prime}\left(u_{-}\right), \quad f\left(u_{+}\right)=f\left(u_{-}\right) .
$$

The last two assumptions guarantee that, when $\varepsilon=0$, the jump from the value $u_{-}$to $u_{+}$is admissible and its speed of propagation, dictated by the Rankine-Hugoniot condition $s \llbracket u \rrbracket=\llbracket f(u) \rrbracket$ is zero. In this case, equation (1.1) with $\varepsilon=0$ possesses a one-parameter family $\left\{U_{\text {hyp }}(\cdot ; \xi)\right\}$ of stationary solutions satisfying the boundary conditions, given by

$$
U_{\text {hyp }}(x ; \xi):=u_{-} \chi_{(-\ell, \xi)}(x)+u_{+} \chi_{(\xi, \ell)}(x),
$$

where $\chi_{I}$ denotes the characteristic function of the set $I$.

For $\varepsilon>0$, the situation is different. The presence of the Laplace operator at the right hand-side of (1.1), together with the boundary conditions $u_{ \pm}$, has the effect of a drastic reduction of the number of stationary solutions: from infinitely many to a single stationary state (see [5]), denoted here by $\bar{U}_{\text {par }}^{\varepsilon}=\bar{U}_{\text {par }}^{\varepsilon}(x)$. As an example, in the case of the Burgers equation, $f(u)=\frac{1}{2} u^{2}$, there holds

$$
\bar{U}_{\mathrm{par}}^{\varepsilon}(x)=-\kappa \tanh \left(\frac{\kappa x}{2 \varepsilon}\right)
$$

where $\kappa=\kappa\left(\varepsilon, \ell, u_{ \pm}\right)$is univocally determined once the boundary conditions are imposed. In the limit $\varepsilon \rightarrow 0^{+}$, the single steady state $\bar{U}_{\text {par }}^{\varepsilon}$ converges pointwise to $\bar{U}_{\text {hyp }}:=U_{\text {hyp }}(\cdot ; 0)$. Moreover, the single steady state $\bar{U}_{\text {par }}^{\varepsilon}(x)$ is asymptotically stable (for more details, see the spectral analysis around the equilibrium performed in [5]), so that, starting from an initial datum close to this equilibrium configuration, the time dependent solution approaches the steady state for $t \rightarrow+\infty$.

Next question is to understand what happens to the dynamics of the solutions to (1.1) generated by initial data still presenting a sharp transition from $u^{-}$to $u^{+}$, but localized far from the position of the steady state $\bar{U}_{\text {par }}^{\varepsilon}$. Numerical computations (see Fig. 1) show that, starting with a decreasing initial datum, a shock layer is formed in a $\mathcal{O}(1)$ time scale, so that the solution is approximately given by a translation of the (unique) stationary solution of the problem. Once such a layer is formed, it moves towards the location corresponding to the equilibrium solution, and this motion is extremely slow.

\section{The Family of APproximate STEAdy STATES AND THE LineARIZED System}

For a rigorous description of the dynamics generated by initial data localized far from the equilibrium, the strategy we use is the following: firstly, we build up a one-parameter family of functions $\left\{U_{\text {par }}^{\varepsilon}(\cdot ; \xi)\right\}_{\xi \in I}$ such that $U_{\text {par }}^{\varepsilon}(\cdot ; \bar{\xi})=\bar{U}_{\text {par }}^{\varepsilon}$ for some $\bar{\xi} \in I$. When $\xi \neq \bar{\xi}$, an element of the family can be seen as an approximate stationary solution to (1.1), in the sense that

$$
\mathcal{F}^{\varepsilon}\left[U^{\varepsilon}\right]:=\varepsilon \partial_{x}^{2} u-\partial_{x} f(u) \rightarrow 0 \quad \text { as } \quad \varepsilon \rightarrow 0
$$

Precisely, we require that $\mathcal{F}^{\varepsilon}\left[U^{\varepsilon}\right]$ belongs to the dual space of the continuous functions space $C(I)$ and that there exists a family of smooth positive functions $\Omega^{\varepsilon}=\Omega^{\varepsilon}(\xi)$, uniformly convergent to zero as $\varepsilon \rightarrow 0$, such that, for any $\xi \in I$, there holds

$$
\left|\left\langle\psi(\cdot), \mathcal{F}^{\varepsilon}\left[U^{\varepsilon}(\cdot, \xi)\right]\right\rangle\right| \leq \Omega^{\varepsilon}(\xi)|\psi|_{L \infty} \quad \forall \psi \in C(I) .
$$

Hence we assume that the error $\mathcal{F}^{\varepsilon}\left[U^{\varepsilon}\right]$, that describes how far is $U^{\varepsilon}$ from being the exact steady state of the problem, has to be small in $\varepsilon$ in the weak sense described in (2.1). 


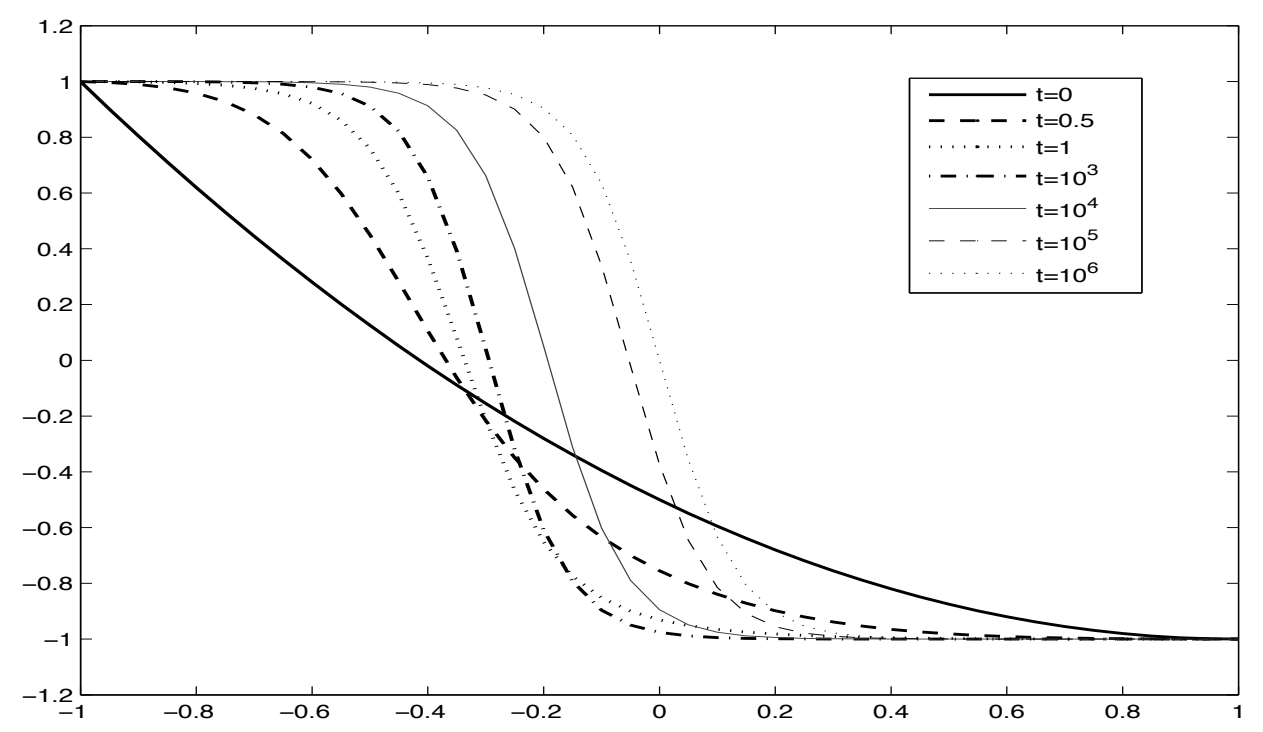

FiguRE 1. The solution to (1.1) with $\varepsilon=0.07, u_{ \pm}=\mp 1$ and $u_{0}(x)=\left(x^{2}-2 x-1\right) / 2$.

Example 2.1. In the case of the Burgers equation, i.e. $f(u)=\frac{1}{2} u^{2}$ and $u^{ \pm}:=\mp u_{*}$, for some $u_{*}>0$, to build up the family of approximate steady states, we consider a function obtained by matching two different steady states satisfying, respectively, the left and the right boundary condition together with the request $U^{\varepsilon}(\xi)=0$; in formulas,

$$
U^{\varepsilon}(x ; \xi)= \begin{cases}\kappa_{-} \tanh \left(\kappa_{-}(\xi-x) / 2 \varepsilon\right) & \text { in }(-\ell, \xi) \\ \kappa_{+} \tanh \left(\kappa_{+}(\xi-x) / 2 \varepsilon\right) & \text { in }(\xi, \ell),\end{cases}
$$

where $\kappa_{ \pm}$are chosen so that the boundary conditions are satisfied

$$
\kappa_{ \pm} \tanh \left(\frac{\kappa_{ \pm}}{2 \varepsilon}(\xi \mp \ell)\right)=u_{ \pm} .
$$

By direct substitution, denoting by $\delta_{x=\xi}$ the usual Dirac's delta distribution concentrated at $x=\xi$, we obtain the identity

$$
\mathcal{F}^{\varepsilon}\left[U^{\varepsilon}(\cdot ; \xi)\right]=\llbracket \partial_{x} U^{\varepsilon} \rrbracket_{x=\xi} \delta_{x=\xi}
$$

in the sense of distributions. In particular, $U^{\varepsilon}(\cdot, \xi)$ is a stationary solution if and only if $\xi=0$. Moreover, it is possible to give the following asymptotic representation

$$
\llbracket \partial_{x} U^{\varepsilon} \rrbracket_{x=\xi}=\frac{2 u_{*}^{2}}{\varepsilon}\left(e^{-u_{*}(\ell+\xi) / \varepsilon}-e^{-u_{*}(\ell-\xi) / \varepsilon}\right)+\text { l.o.t. } \sim C \xi e^{-C / \varepsilon},
$$

showing that, recalling (2.1), the term $\Omega^{\varepsilon}(\xi)$ is null at $\xi=0$ and exponentially small for $\varepsilon \rightarrow 0^{+}$.

In order to describe the dynamics of solutions localized far from the position of the steady state, the second step is the linearization of the original equation (1.1) around an element of the family $\left\{U^{\varepsilon}(x ; \xi)\right\}_{\xi \in I}$. Hence, we look for a solution $u$ in the form

Substituting into (1.1), we obtain

$$
u(\cdot, t)=U^{\varepsilon}(\cdot ; \xi(t))+v(\cdot, t) .
$$

$$
\partial_{t} v=\mathcal{L}_{\xi}^{\varepsilon} v+\mathcal{F}^{\varepsilon}\left[U^{\varepsilon}(\cdot ; \xi)\right]-\partial_{\xi} U^{\varepsilon}(\cdot ; \xi) \frac{d \xi}{d t}+\mathcal{Q}^{\varepsilon}[v, \xi],
$$


where

$$
\begin{aligned}
\mathcal{L}_{\xi}^{\varepsilon} v & :=\varepsilon \partial_{x}^{2} v-\partial_{x}\left(f^{\prime}\left(U^{\varepsilon}(\cdot ; \xi)\right) v\right) \\
\mathcal{Q}^{\varepsilon}[v, \xi] & :=-\partial_{x}\left\{f\left(U^{\varepsilon}(\cdot ; \xi)+v\right)-f\left(U^{\varepsilon}(\cdot ; \xi)\right)-f^{\prime}\left(U^{\varepsilon}(\cdot ; \xi)\right) v\right\}
\end{aligned}
$$

\subsection{Spectral Analysis}

To study properties of the solution $v$ to (2.4), we study the eigenvalue problem for the linearized operator $\mathcal{L}_{\xi}^{\varepsilon}$, that reads

$$
\varepsilon \partial_{x}^{2} \varphi-\partial_{x}\left(f^{\prime}\left(U^{\varepsilon}(\cdot ; \xi)\right) \varphi\right)=\lambda \varphi
$$

complemented with Dirichlet boundary conditions. The first result we state is the following:

Proposition 2.2. The spectrum of the operator $\mathcal{L}_{\xi}^{\varepsilon}$ is composed by a sequence $\left\{\lambda_{k}^{\varepsilon}(\xi)\right\}_{k \in \mathbb{N}}$ of real and negative eigenvalues.

Our second result gives a detailed description of the location of the eigenvalues, under appropriate hypotheses on the family $f^{\prime}\left(U^{\varepsilon}\right)$.

Proposition 2.3. Let $a^{\varepsilon}:=f^{\prime}\left(U^{\varepsilon}\right)$ be a family of functions satisfying the following assumptions.

H0. There exists $C>0$, indipendent on $\varepsilon>0$, such that

$$
\left|a^{\varepsilon}\right|_{\infty}+\varepsilon\left|\frac{d a^{\varepsilon}}{d x}\right|_{\infty} \leq C
$$

H1. The function $a^{\varepsilon}$ is twice differentiable at any $x \neq \xi$ and

$$
\frac{d a^{\varepsilon}}{d x}, \frac{d^{2} a^{\varepsilon}}{d x^{2}}<0<a^{\varepsilon} \quad \text { in }(-\ell, \xi), \quad \text { and } \quad a^{\varepsilon}, \frac{d a^{\varepsilon}}{d x}<0<\frac{d^{2} a^{\varepsilon}}{d x^{2}} \quad \text { in }(\xi, \ell) .
$$

H2. For any $C>0$ there exists $c_{0}>0$ such that, for any $x$ satisfying $|x-\xi| \geq c_{0} \varepsilon$, there hold

$$
\left|a^{\varepsilon}-a^{0}\right|_{L^{1}} \leq C \varepsilon \quad \text { and } \quad \varepsilon\left|\frac{d a^{\varepsilon}}{d x}\right| \leq C .
$$

where $a^{0}:=a_{-} \chi_{(-\ell, \xi)}+a_{+} \chi_{(\xi, \ell)}$ for some $a_{+}<0<a_{-}$.

H3. There exists the left/right first order derivatives of $a^{\varepsilon}$ at $\xi$ and

$$
\liminf _{\varepsilon \rightarrow 0^{+}} \varepsilon\left|\frac{d a^{\varepsilon}}{d x}(\xi \pm)\right|>0
$$

for some $\xi \in(-\ell, \ell)$.

Then there exist constants $C, c>0$ such that

$$
\lambda_{k}^{\varepsilon} \leq-C / \varepsilon \quad \text { and } \quad-e^{-c / \varepsilon} \leq \lambda_{1}^{\varepsilon}<0
$$

for any $k \geq 2$.

For the proof of the Propositions 2.2 and 2.3 we refer to [8, Section 4]. Fore more details, see [8, Proposition 4.1], [8, Proposition 4.4] and [8, Corollary 4.5]. 
Example 2.4. In the case of a Burgers flux, i.e. $f(u)=u^{2} / 2$, it is possible to give an asymptotic expression for the first eigenvalue of the linearized operator $\mathcal{L}_{\xi}^{\varepsilon}$. We have

$$
\lambda_{1}^{\varepsilon} \sim-\frac{u_{*}^{2}}{2 \varepsilon}\left(e^{-u_{*}(\ell-\xi) / \varepsilon}+e^{-u_{*}(\ell+\xi) / \varepsilon}\right) .
$$

Heuristically, the large time behavior of solutions is described by term of the order $e^{\lambda_{1}^{\varepsilon} t}$. As a consequence of (2.6), the convergence towards the equilibrium is exponentially slow, when $\varepsilon$ is small.

\subsection{The projection method}

Let us go back to the equation (2.4) for the perturbation $v$; since we have proved that the first eigenvalue of the linearized operator $\mathcal{L}_{\xi}^{\varepsilon}$ goes to zero in the limit $\varepsilon \rightarrow 0$, denoting by $\phi_{k}^{\varepsilon}=\phi_{k}^{\varepsilon}(\cdot ; \xi)$ and $\psi_{k}^{\varepsilon}=\psi_{k}^{\varepsilon}(\cdot ; \xi)$ the eigenfunctions of the operator $\mathcal{L}_{\xi}^{\varepsilon}$ and of the adjoint operator $\mathcal{L}_{\xi}^{\varepsilon, *}$ respectively, and setting

$$
v_{k}=v_{k}(\xi ; t):=\left\langle\psi_{k}^{\varepsilon}(\cdot ; \xi), v(\cdot, t)\right\rangle
$$

we impose that the first component $v_{1}$ of the perturbation $v$ is identically zero. Precisely, we set an algebraic condition ensuring orthogonality between $\psi_{1}^{\varepsilon}$ and $v$, so that the equation for the parameter $\xi(t)$ is chosen in such a way that the unique growing terms in the perturbation $v$ are canceled out. In formulas

$$
\frac{d}{d t}\left\langle\psi_{1}^{\varepsilon}(\cdot ; \xi(t)), v(\cdot, t)\right\rangle=0 \quad \text { and } \quad\left\langle\psi_{1}^{\varepsilon}\left(\cdot ; \xi_{0}\right), v_{0}(\cdot)\right\rangle=0
$$

Using equation (2.4) and linearizing around $v \sim 0$, we end up with the following coupled system for the perturbation $v$ and the shock layer location $\xi$,

$$
\left\{\begin{array}{c}
\frac{d \xi}{d t}=\theta^{\varepsilon}(\xi)\left(1+\left\langle\partial_{\xi} \psi_{1}^{\varepsilon}, v\right\rangle\right) \\
\partial_{t} v=H^{\varepsilon}(x ; \xi)+\left(\mathcal{L}_{\xi}^{\varepsilon}+\mathcal{M}_{\xi}^{\varepsilon}\right) v
\end{array}\right.
$$

obtained by linearizing with respect to $v$ (so that all the nonlinear terms of order $o(v)$ are neglected) and by keeping the nonlinear dependence on $\xi$, in order to describe the dynamics of the interface far from the equilibrium. In particular

$$
\begin{aligned}
\theta^{\varepsilon}(\xi) & :=\frac{\left\langle\psi_{1}^{\varepsilon}, \mathcal{F}^{\varepsilon}\left[U^{\varepsilon}\right]\right\rangle}{\left\langle\psi_{1}^{\varepsilon}, \partial_{\xi} U^{\varepsilon}\right\rangle}, \\
H^{\varepsilon}(x ; \xi) & :=\mathcal{F}^{\varepsilon}\left[U^{\varepsilon}(x ; \xi)\right]-\partial_{\xi} U^{\varepsilon}(x ; \xi) \theta^{\varepsilon}(\xi), \\
\mathcal{M}_{\xi}^{\varepsilon} v & :=-\partial_{\xi} U^{\varepsilon}(\cdot ; \xi) \theta^{\varepsilon}(\xi)\left\langle\partial_{\xi} \psi_{1}^{\varepsilon}, v\right\rangle .
\end{aligned}
$$

Example 2.5. An interesting term in the equation for the shock layer position is the leading order term $\theta^{\varepsilon}(\xi)$, that heuristically measures the speed of the interface. Indeed, formally, for small $\varepsilon$ and small $v$, the dynamics of the parameter $\xi$ is approximately given by

$$
\frac{d \xi}{d t}=\theta^{\varepsilon}(\xi)+\ldots
$$

In the case of the Burgers equation, i.e. $f(u)=u^{2} / 2$, in order to give an asymptotic expression for the term $\theta^{\varepsilon}$, we need to identify the functions $\psi_{1}^{\varepsilon}$ and $\partial_{\xi} U^{\varepsilon}$ in the limiting regime $\varepsilon \rightarrow 0$, at least approximately. For $\varepsilon \sim 0$, the function $\psi_{1}^{\varepsilon}$ is close to the eigenfunction of the operator $\mathcal{L}_{\xi}^{0, *}$ relative to the eigenvalue $\lambda=0$, with

$$
a^{0}(x ; \xi):=u_{-} \chi_{(-\ell, \xi)}(x)+u_{+} \chi_{(\xi, \ell)}(x) .
$$


Hence, we obtain the representation formula

$$
\psi_{1}^{\varepsilon}(x) \sim \psi_{1}^{0}(x):= \begin{cases}\left(1-e^{u_{+}(\ell-\xi) / \varepsilon}\right)\left(1-e^{-u_{-}(\ell+x) / \varepsilon}\right) & x<\xi, \\ \left(1-e^{-u_{-}(\ell+\xi) / \varepsilon}\right)\left(1-e^{u_{+}(\ell-x) / \varepsilon}\right) & x>\xi,\end{cases}
$$

so that $\psi_{1}^{\varepsilon} \sim 1$. Additionally, with the approximation $U^{\varepsilon}(x ; \xi) \sim U_{\text {hyp }}(x ; \xi)$, defined in (1.3), we expect $\partial_{\xi} U^{\varepsilon}$ to converge to $-\llbracket u \rrbracket \delta_{\xi}$ as $\varepsilon \rightarrow 0$ in the sense of distributions, so that $\left\langle\psi_{1}^{\varepsilon}, \partial_{\xi} U^{\varepsilon}\right\rangle \sim-\llbracket u \rrbracket$. Therefore, we deduce an (approximate) expression for the function $\theta^{\varepsilon}$ that, with the choice of $U^{\varepsilon}$ proposed in Example 2.1, reduces to

$$
\theta^{\varepsilon}(\xi) \sim-\frac{1}{\llbracket u \rrbracket}\left\langle 1, \mathcal{F}\left[U^{\varepsilon}\right]\right\rangle \sim \frac{1}{\varepsilon} u_{*}\left(e^{-u_{*}(\ell+\xi) / \varepsilon}-e^{-u_{*}(\ell-\xi) / \varepsilon}\right),
$$

which coincides with the corresponding formula determined in [10].

\section{The metastable Dynamics}

In this Section we present a result concerning the solutions to the system for the couple $(\xi, v)$

$$
\left\{\begin{aligned}
\frac{d \xi}{d t} & =\theta^{\varepsilon}(\xi)\left(1+\left\langle\partial_{\xi} \psi_{1}^{\varepsilon}, v\right\rangle\right) \\
\partial_{t} v & =H^{\varepsilon}(x ; \xi)+\left(\mathcal{L}_{\xi}^{\varepsilon}+\mathcal{M}_{\xi}^{\varepsilon}\right) v
\end{aligned}\right.
$$

complemented with initial data

$$
\xi(0)=\xi_{0} \in(-\ell, \ell) \quad \text { and } \quad v(x, 0)=v_{0}(x) \in L^{2}(I) .
$$

Before stating our first result, we precise the hypotheses we need on the terms of the system

H1. The family $\left\{U^{\varepsilon}(\cdot, \xi)\right\}$ is such that $\mathcal{F}^{\varepsilon}\left[U^{\varepsilon}\right]$ belongs to the dual space of $C(I)$ and there exists a family of functions $\Omega^{\varepsilon}(\xi)$ such that

$$
\left|\left\langle\psi(\cdot), \mathcal{F}^{\varepsilon}\left[U^{\varepsilon}(\cdot, \xi)\right]\right\rangle\right| \leq \Omega^{\varepsilon}(\xi)|\psi|_{L^{\infty}} \quad \forall \psi \in C(I),
$$

with $\Omega^{\varepsilon}(\xi)$ converging to zero as $\varepsilon \rightarrow 0$, uniformly with respect to $\xi$.

H2. There holds

$$
\Omega^{\varepsilon}(\xi) \leq C\left|\lambda_{1}^{\varepsilon}(\xi)\right|, \quad \forall \xi \in(-\ell, \ell)
$$

for some constant $C>0$ independent on $\varepsilon$ and $\xi$.

H3. Let $\varphi_{k}^{\varepsilon}(\cdot ; \xi)$ and $\psi_{k}^{\varepsilon}(\cdot ; \xi)$ be the sequence of eigenfunction for the operators $\mathcal{L}_{\xi}^{\varepsilon}$ and $\mathcal{L}_{\xi}^{\varepsilon, *}$, respectively. We assume

$$
\sum_{j}\left\langle\partial_{\xi} \psi_{k}^{\varepsilon}, \varphi_{j}^{\varepsilon}\right\rangle^{2}=\sum_{j}\left\langle\psi_{k}^{\varepsilon}, \partial_{\xi} \varphi_{j}^{\varepsilon}\right\rangle^{2} \leq C \quad \forall k
$$

for some constant $C$ independent on the parameter $\xi$.

Remark 3.1. Let us stress that hypotheses $\mathbf{H 1}$ and $\mathbf{H 2}$ are satisfied in the case of the Burgers equation $f(u)=$ $u^{2} / 2$ (see the asymptotic expressions for $\Omega^{\varepsilon}$ and $\lambda_{1}^{\varepsilon}$ determined in (2.3) and (2.6)).

For later use, we use the notation $\Lambda_{k}^{\varepsilon}:=\sup _{\xi \in I} \lambda_{k}^{\varepsilon}(\xi)$.

Theorem 3.1 (Estimate for the perturbation). Let hypotheses $\mathbf{H 1 - 2 - 3}$ be satisfied. Then, denoted by $(\xi, v)$ the solution to the initial-value problem (3.1)-(3.2), for any $\varepsilon$ sufficiently small, there exists a time $T^{\varepsilon}$ of order $\left.|| \Omega^{\varepsilon}\right|_{L^{\infty}} ^{-1} \ln \left|\Omega^{\varepsilon}\right|_{L^{\infty}} \mid$ such that for any $t \leq T^{\varepsilon}$ the solution $v$ can be represented as $v=z+R$ where $z$ is defined by

$$
z(x, t):=\sum_{k \geq 2} v_{k}(0) \exp \left(\int_{0}^{t} \lambda_{k}^{\varepsilon}(\xi(s)) d s\right) \varphi_{k}^{\varepsilon}(x ; \xi(t)),
$$


and the remainder $R$ satisfies the estimate

$$
|R|_{L^{2}} \leq C\left|\Omega^{\varepsilon}\right|_{L^{\infty}}\left\{e^{\Lambda_{1}^{\varepsilon} t}\left|v_{0}\right|_{L^{2}}+1\right\}
$$

for some constant $C>0$.

Estimate (3.5) can be used to decouple system (3.1); indeed, the function $\xi$ satisfies

$$
\frac{d \xi}{d t}=\theta^{\varepsilon}(\xi)(1+r) \quad \text { with } \quad|r| \leq C\left(\left|v_{0}\right|_{L^{2}} e^{\Lambda_{2}^{\varepsilon} t}+\left|\Omega^{\varepsilon}\right|_{L^{\infty}}\right),
$$

so that, if $\varepsilon$ and $\left|v_{0}\right|_{L^{2}}$ are sufficiently small, it is possible to prove the following Theorem characterizing the metastable behavior of the solution through the slow motion of the shock layer location.

Theorem 3.2 (Slow motion of the shock layer). Let hypotheses H1-2-3 be satisfied. Assume also

$$
\xi \theta^{\varepsilon}(\xi)<0 \quad \text { for any } \xi \in I, \xi \neq 0 \quad \text { and } \quad \theta^{\varepsilon^{\prime}}(\bar{\xi})<0 .
$$

Then for $\varepsilon$ and $\left|v_{0}\right|_{L^{2}}$ sufficiently small, the solution $\xi(t)$ converges to $\bar{\xi}$ as $t \rightarrow+\infty$.

Proof. Thank to assumption H1, for $\varepsilon$ and $\left|v_{0}\right|_{L^{2}}$ sufficiently small, estimate (3.5) holds. Hence, for any initial datum $\xi_{0}$, the variable $\xi=\xi(t)$ satisfies (3.6), and, as a consequence, it converges exponentially fast to $\bar{\xi}$ as $t \rightarrow+\infty$.

Corollary 3.3. Let the hypotheses of Theorem 3.3 be satisfied, then

$$
|\xi(t)-\bar{\xi}| \leq\left|\xi_{0}\right| e^{\beta^{\varepsilon} t}, \quad \beta^{\varepsilon} \sim \theta^{\varepsilon \prime}(\bar{\xi})
$$

where $\theta^{\varepsilon^{\prime}}(\bar{\xi}) \rightarrow 0$ as $\varepsilon \rightarrow 0$.

Proof. From (3.5), using a standard method of separation of variables, we get

$$
\int_{\xi_{0}}^{\xi} \frac{d \xi}{\theta^{\varepsilon}(\xi)}=\int_{0}^{t} d t
$$

Hence, since $\theta^{\varepsilon}(\xi) \sim \theta^{\varepsilon^{\prime}}(\bar{\xi})(\xi-\bar{\xi})$, by integrating we end up with (3.7).

For the proof of Theorem 3.1, we refer to [8, Theorem 2.1].

Estimate (3.7) shows the slow motion of the shock layer location: precisely, the evolution of the interface towards its equilibrium is much slower as $\varepsilon$ becomes smaller. For example, in the case of the Burgers equation, $\beta^{\varepsilon} \sim-e^{-1 / \varepsilon}$, so that this motion is indeed exponentially slow.

These rigorous results are also validated numerically: the following table shows a numerical computation for the location of the shock layer for different values of the parameter $\varepsilon$ and $f(u)=u^{2} / 2$. The initial datum is given by $u_{0}(x)=\frac{1}{2} x^{2}-x-\frac{1}{2}$. We can see that the convergence towards $\bar{\xi}=0$ is slower as $\varepsilon$ becomes smaller. 
The numerical location of the shock layer $\xi(t)$ for different values of the parameter $\varepsilon$

\begin{tabular}{|c|c|c|c|c|}
\hline TIME $t$ & $\xi(t), \varepsilon=0.1$ & $\xi(t), \varepsilon=0.07$ & $\xi(t), \varepsilon=0.055$ & $\xi(t), \varepsilon=0.04$ \\
\hline \hline 0.2 & -0.3954 & -0.4010 & -0.4037 & -0.4065 \\
\hline 1 & -0.3310 & -0.3364 & -0.3384 & -0.3808 \\
\hline $10^{2}$ & -0.2546 & -0.3240 & -0.3300 & -0.3315 \\
\hline $10^{3}$ & -0.0924 & -0.2944 & -0.3284 & -0.3315 \\
\hline $10^{4}$ & $-1.0583 * 10^{-4}$ & -0.1931 & -0.3141 & -0.3312 \\
\hline $10^{6}$ & $-4.3033 * 10^{-12}$ & $-8.7386 * 10^{-6}$ & -0.1424 & -0.3288 \\
\hline
\end{tabular}

\section{REFERENCES}

[1] Alikatos N.D., Bates P.W., Fusco G. (1991). Slow motion for the Cahn-Hilliard equation in one space dimension J. Differential Equations 90, no. 1, 81-135

[2] Berestycki, H.; Kamin S.; Sivashinsky G.; Metastability in a flame front evolution equation Interfaces Free Bound. 3 (2001), no. 4, 361-392.

[3] Carr J., Pego R. L. (1989). Metastable patterns in solutions of $u_{t}=\epsilon^{2} u_{x x}-f(u)$, Comm. Pure Appl. Math. 42, no. 5, 523-576.

[4] Fusco G., Hale J. K. (1989). Slow-motion manifolds, dormant instability, and singular perturbations, J. Dynam. Differential Equations 1, no. 1, 75-94.

[5] Kreiss, G., Kreiss, H. (1986). Convergence to steady state of solution of Burgers equation Appl. Numer. Math. 2, no. 3-5, $161-179$.

[6] Laforgue J.G.L., O'Malley Jr R.E. (1994). On the motion of viscous shocks and the supersensitivity of their steady-state limits, Methods Appl. Anal. 1, no. 4, 465-487.

[7] Laforgue J.G.L., O'Malley Jr R.E. (1995). Shock layer movement for Burgers equation, Perturbations methods in physical mathematics (Troy, NY, 1993). SIAM J. Appl. Math. 55, no. 2, 332-347.

[8] Mascia C., Strani M. (2012). Metastability for nonlinear parabolic equations with application to scalar viscous conservation laws, SIAM J. Math. Anal., to appear.

[9] Pego R.L. (1989). Front migration in the nonlinear Cahn-Hilliard equation, Proc. Roy. Soc. London Ser. A 422, no. 1863, 261-278.

[10] Reyna L.G., Ward M.J. (1995). On the exponentially slow motion of a viscous shock, Comm. Pure Appl. Math. 48, no. 2, $79-120$.

[11] Strani M., Slow motion of internal shock layers for the Jin-Xin system in one space dimension, submitted.

[12] Sun X., Ward M.J. (1999). Metastability for a generalized Burgers equation with applications to propagating flame fronts, European J. Appl. Math. 10, no. 1, 27-53. 